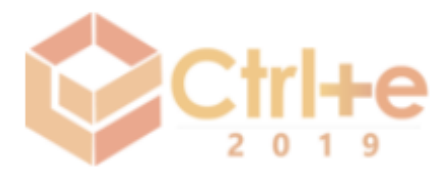

IV Congresso sobre Tecnologias na Educação (Ctrl+E 2019)

Recife, Pernambuco - Brasil

28 a 30 de agosto de 2019

\title{
Programando a Várias Mãos: um Relato de Experiência da Construção Colaborativa de Algoritmos
}

\author{
Aladir Ferreira da Silva Júnior1, Leizer Fernandes Moraes1, Rita Rodrigues de \\ Souza1
}

1 Instituto Federal de Educação, Ciência e Tecnologia de Goiás (IFG) - Câmpus Jataí

Núcleo de Informática na Educação (NINE) - 75.804-714 - Jataí - GO - Brasil

\{leizer.moraes, aladir.junior, rita.souza\}@ifg.edu.br

\begin{abstract}
This study consists in a description of experience of using Google Docs in educational practice. Scientific research has shown that there are barriers in building learning for college students, especially in developing of their computer programming ability. The fundamental contents for the accomplishment of this activity may not be presented in a way that favors learning. Following the Collaborative Learning approach, a problem-solving activity mediated by Google Docs with three initial classes of higher education was applied. It has been shown possible to verify that in the teaching-learning of Algorithms, Google Docs presents encouraging results.
\end{abstract}

Resumo. Neste artigo se compartilha um relato de experiência com o uso do Google Docs em prática educativa. Pesquisas cientificas têm mostrado que há barreiras na construção do aprendizado de alunos de cursos superiores, especialmente quanto ao desenvolvimento da habilidade de se programar computadores. Os conteúdos fundamentais para a realização dessa atividade talvez não estejam sendo apresentados de modo a favorecer o aprendizado. Seguindo o proposto na abordagem da Aprendizagem Colaborativa, aplicou-se uma atividade de resolução de problemas mediada pelo Google Docs com três turmas iniciais do ensino superior e foi possivvel constatar que no auxílio ao ensino-aprendizagem de Algoritmos, este apresenta resultados promissores.

\section{Introdução}

Há algum tempo persistem índices consideráveis de reprovação nas disciplinas iniciais voltadas à programação de computadores, bem como de evasão de cursos superiores da área de Computação. Relatos de que estas disciplinas têm conteúdo árido [Oliveira e Nogueira 2015; Machado 2016], de que a forma como esse conteúdo é trabalhado pode não ser adequada [Borges, 2000; Fontes e Silva, 2008; Oliveira e Nogueira 2015] e causar desmotivação [Rodrigues 2004] são constatados em simples busca na Web.

Estudos compilados de 2001 a 2014, por Ramos et al. [2015], evidenciam que no Brasil, o índice de reprovação em matérias sobre o ensino de programação e algoritmos para iniciantes do ensino superior é em média 45,6\%, podendo após apresentar intervenções na estratégia de ensino, sobretudo nas estratégias mais tradicionais, ter uma redução de aproximadamente $13 \%$ nesse mesmo índice. Considera-se, no entanto, que um índice $32,6 \%$ de reprovação não seja aceitável e deve-se a todo custo buscar estratégias para a mitigação desse importante problema. 
Muitos são os esforços já aplicados no ensino inicial de Algoritmos com o uso de tecnologias e estratégias inovadoras [Rapkiewicz et al. 2006; Barcelos, Tarouco e Bercht 2009; Priesnitz Filho, Abegg e Simonetto 2012; Salgado; Castro; Castro 2013; Silva, Tedesco e Melo 2014; Machado 2016], relatando resultados promissores. Deve-se levar em consideração que grande parte dos alunos que adentram o ensino superior não vêm com uma formação em fundamentos de programação, lógica de programação, raciocínio lógico/matemático, conhecimentos fundamentais para a programação de computadores.

A qualidade da mediação desenvolvida pelo professor em sala de aula ou no laboratório de informática pode ser um diferencial no ensino-aprendizagem dessa disciplina e, assim, a inclusão de tecnologias e estratégias inovadoras de ensino deve ser fomentada. Isso motivou o desenvolvimento dessa pesquisa que propõe uma abordagem para o ensino-aprendizagem de algoritmos, especialmente quanto à construção destes em laboratório de informática de modo colaborativo usando o Google Docs.

O Google Docs é um editor síncrono desenvolvido pela Google que funciona diretamente em um navegador de Internet a partir de um serviço de armazenamento em nuvem (Google Drive). Apesar de ter sido agrupado junto a outros serviços no Google Drive, usaremos o termo Google Docs neste artigo, pois a própria empresa ainda o considera como um serviço que pode ser acessado diretamente (docs.google.com).

\section{Aprendizagem Colaborativa}

Aprendizagem colaborativa é uma abordagem de ensino-aprendizagem em que os discentes se organizam em grupos para resolver um problema, realizar uma tarefa ou criar um produto [Laal 2011]. Nessa abordagem, os grupos devem ser pequenos e seus integrantes devem interagir ativamente entre si, com a coordenação do professor. A interação entre os membros do grupo inclui conversas face a face, discussões em ambientes virtuais (no caso desta pesquisa no bate-papo do Google Docs) e também discussões em um mesmo ambiente físico com o apoio computacional. [Machado 2016]

O relato de Machado [2016] traz que, onde essa abordagem é aplicada, o processo de os alunos apenas ouvirem e fazerem os exercícios dá lugar a discussões em grupos e os estudantes trabalham ativamente com o material do curso. A dinâmica proporcionada pelo Google Docs, confere ao processo de ensino-aprendizagem uma outra dimensão, onde os atores têm que assumir novos papéis para a construção de conhecimentos. A interação e a interlocução entre os participantes do processo são favorecidas não só na questão espaço-tempo, mas sobretudo na possibilidade de construção coletiva e colaborativa de conhecimentos e solução de problemas.

A colaboração suportada no contexto computacional envolve três elementos: comunicação, coordenação e cooperação. Segundo [Pimentel et al. 2006], a comunicação se dá por meio da troca de mensagens; a coordenação se realiza por meio do gerenciamento de pessoas, atividades e recursos; e a cooperação se dá por meio de operações num espaço compartilhado de execução das tarefas. Pode-se perceber que são três elementos distintos, mas que mantêm uma inter-relação centrada na colaboração.

Diversos modelos foram propostos para explicitar a relação entre esses três elementos: modelo 3C, modelo Clover, modelo de colaboração de Murphy entre outros. Neste artigo, discute-se, com mais detalhes, o modelo 3C, tema da próxima seção. 


\subsection{Modelo 3C de Colaboração}

O Modelo 3C, cujas bases referenciam o artigo de Ellis et al. [1991], tem se prestado não apenas a classificar o suporte computacional à colaboração, mas também para avaliar sistemas colaborativos, avaliar sistemas de coautoria, e também para utilizá-lo como base para o desenvolvimento arquitetural de aplicações colaborativas [Pimentel et al. 2006]. Essa gama de aplicações do modelo $3 \mathrm{C}$ nos levou a defini-lo para uso nesse trabalho.

A pesquisa retratada neste artigo partiu do princípio que o $3 \mathrm{C}$ tem potencial para classificar editores colaborativos em nuvem, como é o caso do Google Docs e também pode orientar o processo de análise das atividades desenvolvidas na estratégia que aqui se apresenta, especialmente quanto aos três pilares do trabalho colaborativo, a saber: a comunicação, a coordenação e a cooperação.

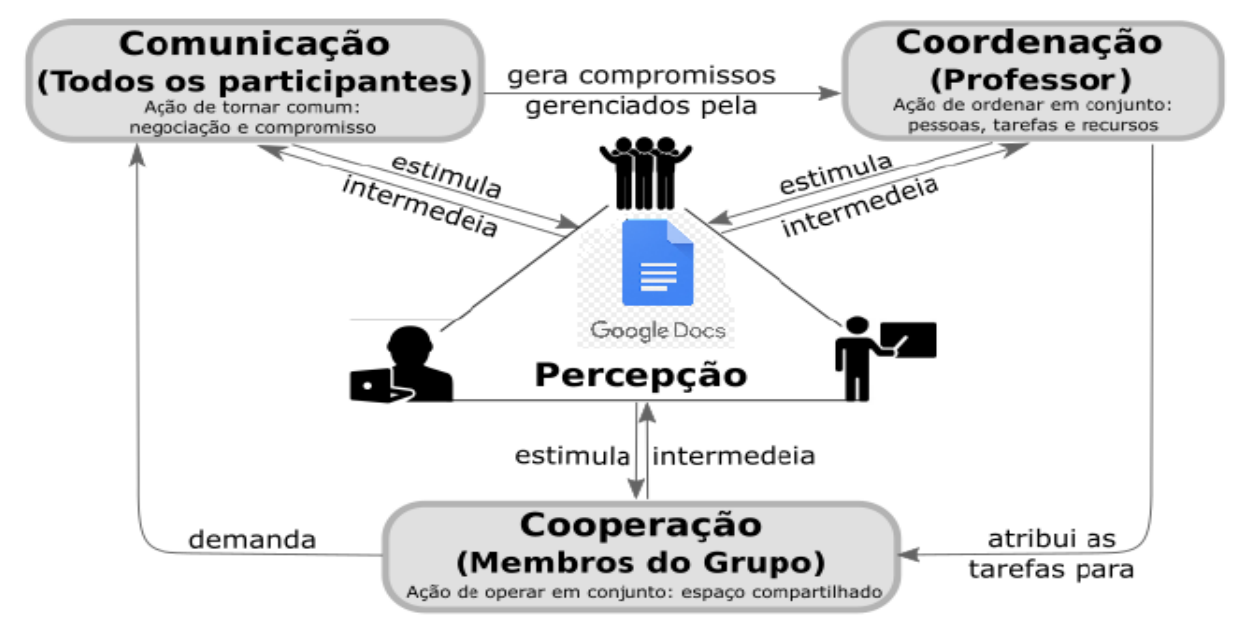

Figura 1 - Google Docs no Modelo 3C de colaboração. Adaptada de [Moura 2017]

$\mathrm{Na}$ Figura 1, se mostram os três pilares da aprendizagem colaborativa apresentados no Modelo 3C, explicitando que o Google Docs se posiciona de modo central, na união, favorecendo as ações de comunicar, de co(ordenar) e de co(operar) entre os membros envolvidos. Enfatiza-se que essa Tecnologia Digital de Informação e Comunicação (TDIC) possibilita o intercâmbio de papéis no processo de ensinoaprendizagem. Professor e aluno compartilham a atuação. O professor, com isso não deixa de exercer sua função, de ter sua responsabilidade no processo formativo, não "perde poder". Ele divide e gerencia as tarefas e responsabilidades. O aluno, por sua vez, sai da zona de conforto de ser ouvinte e passa a atuar, tomar decisões, compartilhar o conhecimento, aprender e ensinar com outro.

\subsection{Trabalhos correlatos}

Apresentação de ferramentas com o intuito de facilitar a aprendizagem em programação de computadores tem sido foco de diversos pesquisadores. [Karavirta et al. 2012; Ihantola et al. 2013; Brandão et al. 2014 e Machado et al. 2018] são alguns exemplos.

A ferramenta Google Docs, no que tange à produção de textos de forma colaborativa, foi tratado em Oliveira e Moraes [2013]; no acompanhamento e avaliação por Costa et al. [2016], ou até mesmo levantando as possibilidades de seu uso como nos estudos de Moi et al. [2016] e Oliveira et al. [2017]. Não se encontrou, até a escrita deste 
artigo, relatos de pesquisas sobre o uso do Google Docs no ensino-aprendizagem de algoritmos, o que fortalece a contribuição da abordagem aqui apresentada.

Julga-se que quanto mais se oferecer ao professor possibilidades e estratégias de aplicação de TDIC no ensino-aprendizagem melhor será para o desenvolvimento do processo, haja vista a fluidez no uso destas pelos atuais discentes e pela diversidade de ferramentas já disponíveis e de novas que surgem a cada dia. $\mathrm{Na}$ próxima seção são apresentados os procedimentos metodológicos utilizados na pesquisa.

\section{Metodologia}

Baseando no recomendado pela Aprendizagem Colaborativa, o professor estabeleceu a divisão da classe de alunos em equipes pequenas, com o número máximo de 3 (três) alunos. Para cada grupo foi proposto um problema a ser resolvido dentro de um tempo de aula (45 minutos). Foi escolhido um tema para os problemas: Estruturas Condicionais. Esse tema foi escolhido por estar presente no ementário de todas as disciplinas que se propõem a apresentar aos alunos os conceitos iniciais de programação de computadores. $\mathrm{O}$ experimento foi realizado com três turmas de alunos de cursos superiores de uma instituição federal de ensino.

\subsection{Sobre a amostragem}

A amostra foi composta por 75 (setenta e cinco) alunos de cursos superiores, sendo 23 (vinte e três) alunos do $1^{\circ}$ período do Curso Superior de Tecnologia em Análise e Desenvolvimento de Sistemas (CSTADS), 27 (vinte e sete) alunos do $1^{\circ}$ período do Curso Superior de Bacharelado em Engenharia Elétrica (CSBEE) e 25 (vinte e cinco) alunos do $2^{\circ}$ período do Curso Superior de Bacharelado em Engenharia Civil (CSBEC). Usando um modelo não-probabilístico foi feita uma Amostragem por Conveniência, haja vista a facilidade de acesso do pesquisador aos alunos, em seu local de trabalho.

\subsection{Descrição e sequência do experimento}

Inicialmente se aplicou um questionário individual para o levantamento de informações de caracterização da própria amostra e também ligadas ao conhecimento da tecnologia que se propunha a aplicar.

O professor dividiu cada uma das três turmas em grupos de no mínimo dois e no máximo três discentes. Para cada grupo, foi lançado um desafio que constituía em resolver por meio da criação de um algoritmo em linguagem de programação $\mathrm{C}$ um problema acerca da temática escolhida para o experimento, a saber: Estruturas Condicionais, comumente chamadas de Estruturas de Decisão. Após a criação do código-fonte os alunos exportavam-no em formato texto puro e usavam esse arquivo para compilação e execução em uma IDE padrão instalada no laboratório.

Achou-se dispensável ministrar treinamento do Google Docs para os alunos, haja vista que eles já dominam as ferramentas disponíveis em suítes de escritório para desenvolverem seus trabalhos escolares e outras atividades cotidianas e também pelo fato de que o Google Docs possui interface similar a essas suítes. Para cada turma, foi realizada uma sessão, em laboratório de informática com 30 computadores com Internet (vide Figura 1), de uso da TDIC para a solução do desafio proposto a cada grupo. 
Cada sessão teve a duração total de 1 hora e 30 minutos, tempo disponível para responder os questionários e resolver o problema. No início da sessão foi oportunizado um tempo de 15 minutos para que aqueles discentes que não possuíam e-mail na plataforma Google pudesse criá-lo, já que essa é uma exigência para o uso dessa TDIC.

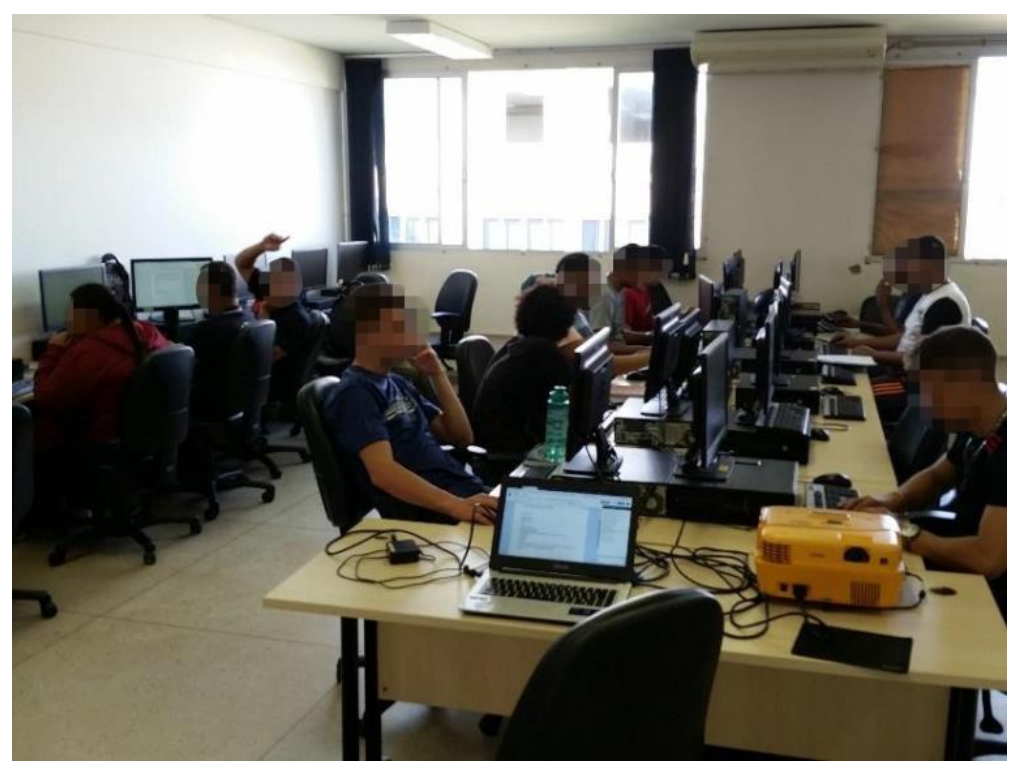

Figura 1: Uma sessão de aplicação do experimento em laboratório.

Aplicou-se um segundo questionário objetivando verificar as percepções da amostra em relação à TDIC empregada nas sessões. A partir dos dados dos questionários e das percepções do professor-observador foi realizada uma análise explicitada na próxima seção.

\section{Resultados e discussões}

As respostas fornecidas por meio dos dois questionários aplicados à amostra foram analisadas em dois eixos: um eixo ligado à caracterização da amostra, ao qual se chamará de Eixo de Caracterização (EC), com dados tais como idade e curso, e outro eixo, ligado às respostas quanto ao uso do Google Docs, ao qual se chamará de Eixo de Uso (EU), com dados relativos às atividades e percepções do aluno ao usar a TDIC.

Inicialmente, procedeu-se a uma análise em relação ao conhecimento da TDIC pela amostra no EU e os resultados indicaram que 60 alunos já conheciam o Google Docs. Isso corresponde a parte significativa da amostra (80\%), o que provocou uma análise em relação ao EC da amostra analisada. Assim no EC, por meio do item "Idade", usando um limiar etário, conforme o conceito preconizado por [Prensky 2001], concluiu-se que 57 (76\%) são considerados nativos digitais, sendo que 52 (mais de 90\%) destes disseram conhecer a tecnologia de aplicativos em nuvem. Despertou-se em conhecer em que medida a amostra se utiliza dessa TDIC, cujos dados agrupados em EU são explicitados na Tabela 1 .

Considerou-se a gradação para Frequência de Uso: Nunca (0), Raramente (1 a 2 vezes/ano), Às vezes (6 vezes/ano), Frequentemente (1 vez ao mês) e Sempre (1 ou mais vezes/semana). Avaliando os dados da Tabela 1, pode-se considerar que os discentes, apesar de conhecerem a TDIC a usam de forma tímida, 59 alunos (78,7\% da amostra), a usa cada dois meses. Quando questionados sobre se já tinham utilizado o Google Docs 
para aprender Algoritmos ou tomado conhecimento de alguma ação assim todos os alunos responderam negativamente.

\begin{tabular}{|l|r|}
\hline \multicolumn{1}{|c|}{ Frequência de uso } & \multicolumn{2}{c|}{$\%$ Alunos } \\
\hline Nunca & $\mathbf{9 , 3}$ \\
\hline Raramente & $\mathbf{8 , 0}$ \\
\hline Às vezes & $\mathbf{7 8 , 7}$ \\
\hline Frequentemente & 2,7 \\
\hline Sempre & $\mathbf{1 , 3}$ \\
\hline
\end{tabular}

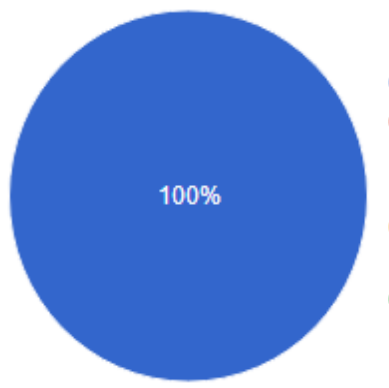

Gráfico 1: Contribuição em \% do Google Docs
Contribui para a aprendizagem

Contribui às vezes para a

aprendizagem, mas é divertido e empolgante

Não gostei dela e acredito que não contribua para 0 aprendizado

Não participei do experimento em laboratório com o uso do Google Docs

Tabela 1 - Uso em \% do Google Docs

Ao mesmo tempo, $100 \%$ da amostra respondeu que considera importante que professores e alunos usem essa tecnologia em sala de aula e fora dela para o aprendizado de lógica de programação, o que corrobora nossa hipótese de que essa TDIC possa contribuir no sentido de dinamizar o ensino-aprendizagem de lógica de programação, algoritmos e disciplinas correlatas. Os alunos também responderam unanimemente que o uso dessa TDIC com essa abordagem contribui para sua aprendizagem, conforme se mostra no Gráfico 1. Além de resultados numéricos agrupados, foram analisados relatos dos participantes do experimento com o uso do Google Docs, como apresentado a seguir.

\subsection{Relato/Reflexão sobre experiência de ensino-aprendizagem de Algoritmos por meio da ferramenta Google Docs}

Durante as sessões do experimento de uso da ferramenta Google Docs, ficou explícita a motivação dos alunos em executar as tarefas propostas. Por meio de observação participante, o professor-pesquisador pode verificar reações diversas sendo a de maior destaque, a identificação de cada elemento do grupo apresentada de uma cor diferente na ferramenta. Apesar de isso gerar algum tipo de brincadeira inicialmente, com o passar dos primeiros 10 minutos se transformou em responsabilidades já que cada um dos discentes queria fazer uma parte do algoritmo proposto.

A visualização simultânea do mesmo código sendo construído, alterado, também gerou euforia inicial gerando comentários do tipo: "que top!", "não sabia que havia isso aqui!". Tais emoções foram catalisadas quando professor fomentou o uso do bate-papo disponível na TDIC (vide Figura 2). Acredita-se que esse sentimento de motivação tenha sido verdadeiro haja vista que os grupos continuaram a usar o Google Docs em casa, chegando a refinar o algoritmo inicialmente feito no laboratório de informática.

Vale ressaltar que o uso da TDIC foi além do experimento e tende a se firmar como uma opção para os discentes, pois relataram estar utilizando o Google Docs para a realização em grupo de tarefas escolares diversas, inclusive projetos finais de disciplinas, em que cada um pode dar sua contribuição para o trabalho independente de horário e local que esteja. Buscando avaliar do quê e como os discentes se beneficiaram ao usar a TDIC, foi proposta a seguinte questão no segundo questionário: Após o uso em laboratório como ferramenta para permitir a criação em grupo do Algoritmo determinado, quais foram suas percepções acerca do uso desta tecnologia em sala de aula? A partir das respostas variadas, fez-se um agrupamento para fins de análise. Os trechos entre aspas estão transcritos em sua forma original. 


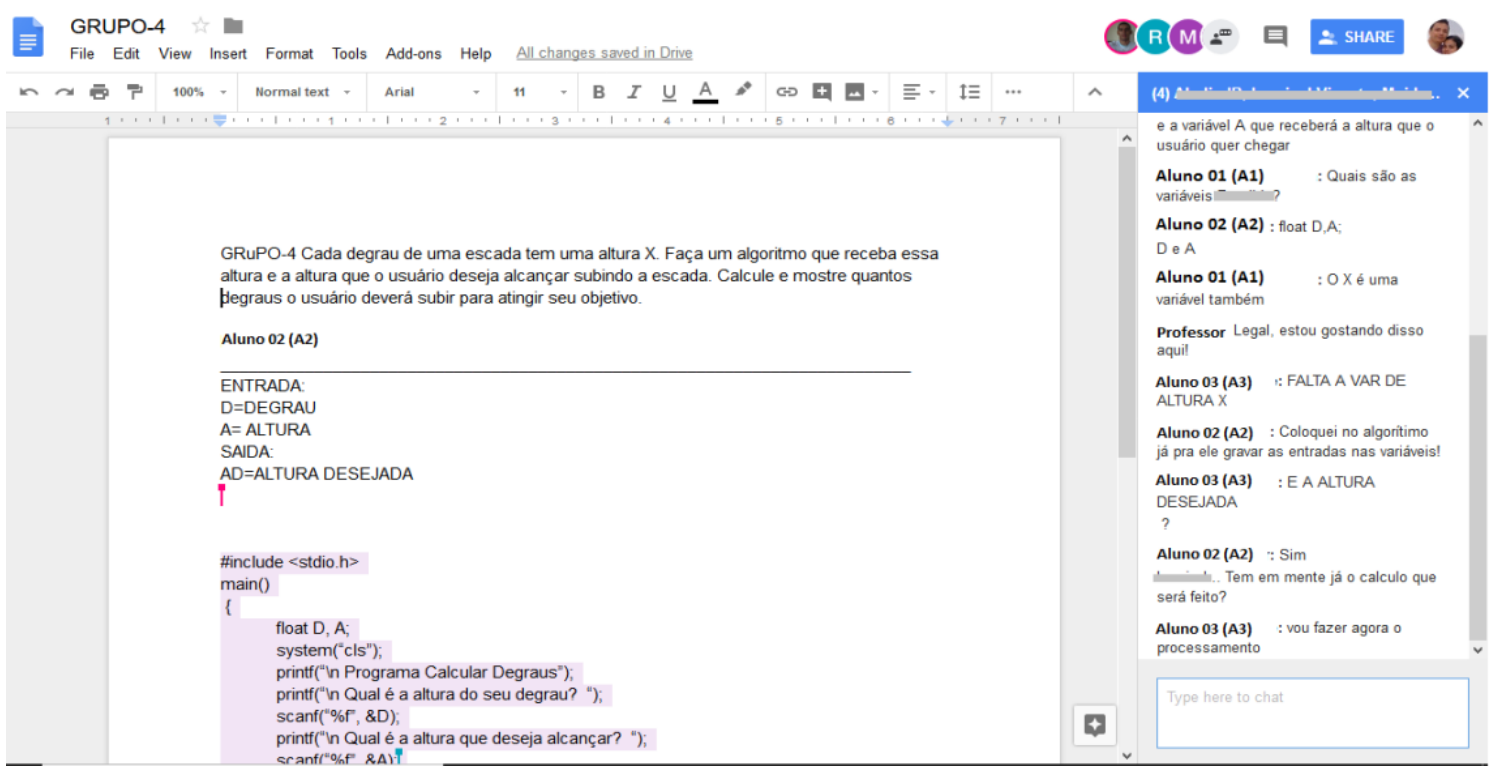

Figura 2: Interações entre os membros de um grupo no ambiente Google Docs.

Foi possível observar pelo relato: (A1) "Achei bem mais prático, porque assim fica mais fácil tirar as dúvidas.”, que a TDIC pode auxiliar suprimindo a inibição do aluno em tirar suas dúvidas. Talvez A1 tenha tido mais facilidade em tirar suas dúvidas junto aos seus pares ou, ainda, por algum auxílio do professor via bate-papo da TDIC.

A colaboração e facilitação do trabalho em grupo foi o quesito mais observado e relatado pelos participantes em relação ao Google Docs: (A3) "Um bom aproveitamento do trabalho em grupo para aprendizagem do conteúdo. Além de ter sido uma experiência divertida.", (A4) "Melhora o desempenho ao trabalhar em grupo", (A5) "Facilita o trabalho em grupo e melhora o desenvolvimento do trabalho em questão", (A7) "Se torna mais fácil a comunicação do trabalho em equipe. Contribui muito para a aprendizagem.", (A8) "Ótimo método, contribuindo com a interação em grupo com todos da turma.", (A9) "Forma de trabalho prática e de fácil utilização. Espetacular ferramenta para se trabalhar em grupo sem a necessidade de estarem reunidos.", (A36) "Trabalhar com essa ferramenta foi muito importante para trabalhar em equipe e trocar experiência com os colegas acompanhamento do professor e com a possibilidade de trabalhar fora da sala de aula." e (A42) "A interação com grupos de trabalho ou estudo ficam mais dinâmicas, conseguimos conversar, trocar ideias e produzir ao mesmo tempo, com isso o compartilhamento de conhecimento fica maior e consequentemente a nossa aprendizagem evolui de modo considerável."

Para se obter dos participantes que características foram mais apreciadas no uso da TDIC, foi proposta a seguinte questão: O que você mais gostou no Google Docs? Alguma característica especial? A partir das respostas, foram identificadas características/recursos tais como (i) diversidade de recursos, como relatado por A3 "Tem muitos recursos que pode utilizar no aprendizado, inclusive trabalhos e slides"; (ii) facilidade de uso, como relatado por A5 "Fácil acesso entre os integrantes do grupo", A7 "Facilidade para fazer trabalhos em grupo" e A48 "Facilidade em compartilhar com várias pessoas"; (iii) compartilhamento e armazenamento facilitados, como é possível ver nos relatos de A8 "O fato de que pode ser compartilhado e usado ao mesmo tempo por várias pessoas", A27 "Poder compartilhar os arquivos e o salvamento automático." e A32 "não é necessário salvar e depois enviar para outra pessoa". 
Adicionalmente, figuram como características apreciadas o (iv) feedback visual e comunicação, como relatado por A22 "A possibilidade de interagir com os participantes através do chat e ainda poder ver em tempo real as alterações sendo realizada por todos.", A6 "poder trabalhar em uma área compartilhada onde todos podem estar editando" e A42 "O fato de que todas as edições podem ser acompanhadas em tempo real, qualquer edição todos do grupo acompanham sem que ninguém fique para trás ou sem compreender o que está sendo feito."; (v) portabilidade, como é possível observar pelo relato de A33 "Dá pra ser usado de qualquer lugar, mesmo que as pessoas não estejam próximas." e; (vi) interatividade, como dito por A19 "Porque a comunicação via redes sociais como é comumente feito, as vezes deixa a desejar, o Google docs vem com essa pegada de interatividade aliada aos estudos". Apenas uma limitação da TDIC foi apontada por A25 referente ao bate-papo: "As mensagens do bate papo apagam se você sai, gostaria q elas permanecessem lá após a saída e que alguém que não estava presente possa ver as mensagens quando entrar.”.

\section{Considerações Finais}

Relatar uma experiência de ensino-aprendizagem acerca da criação de algoritmos de forma colaborativa com o uso do Google Docs foi o foco deste artigo. Essa experiência foi aplicada a três turmas iniciais de cursos superiores de uma instituição federal de ensino. Considera-se que o trabalho de pesquisa realizado teve sua relevância em importantes aspectos, a saber: (i) A inexistência até o momento de relatos de experiência com a tecnologia Google Docs para o ensino-aprendizagem de algoritmos, colaborando assim para o campo de pesquisas da Informática na Educação; (ii) A análise e discussão realizada apontou que apesar do conhecimento dos discentes acerca da existência da tecnologia Google Docs ou similar, essa não é utilizada frequentemente e, portanto, os discentes não costumam usufruir dos benefícios que ela pode trazer quando usada de modo coordenado em sala de aula ou laboratório em favor do ensino-aprendizagem.

Ressalta-se que a adoção da TDIC contribuiu para que os alunos encarassem a tarefa da construção do algoritmo como um desafio a ser superado e, colaborativamente enxergaram-se como equipe para apresentarem uma solução. Apesar de uma certa rivalidade, pelo fato de que é possível verificar quem contribuiu mais utilizando a ferramenta Histórico do Google Docs, todos os grupos atingiram o objetivo da equipe em construir seu respectivo algoritmo em linguagem $\mathrm{C}$.

Encontrar formas mais adequadas de se medir e analisar um diferencial de motivação entre os alunos com a técnica aqui aplicada e os que não passaram pela aplicação da técnica de escrita colaborativa de algoritmos é um objetivo a ser perseguido em pesquisas futuras. Um próximo passo dessa pesquisa é a proposição de uma arquitetura pedagógica para a escrita colaborativa de Algoritmos.

Considera-se a experiência aqui relatada como motivadora, uma vez que foram constatados avanços ao usarmos uma metodologia não comum nas aulas de Algoritmos e correlatas. Ressalta-se ainda a incorporação da TDIC Google Docs na prática dos discentes, pois a estão utilizando para outras finalidades em sua vida acadêmica, o que por si só reveste de importância e relevância a pesquisa realizada e aqui relatada. Por fim, conclui-se que o fato de que alguém tenha conhecimento da existência de uma tecnologia não necessariamente leva esta pessoa a usá-la, mesmo que esse alguém seja considerado 
um nativo digital. Cabe ao professor instigar, incentivar e fomentar o uso de tais tecnologias, incorporando-as à sua prática.

\section{Referências}

Barcelos, R.; Tarouco, L.; Bercht, M (2009). "O uso de mobile learning no ensino de algoritmos”. In: RENOTE - Revista Novas Tecnologias na Educação, 2009. v. 7, n. 3, p. 327-337, 2009.

Borges, M. (2000). "Avaliação de uma metodologia alternativa para a aprendizagem de programação". In Anais do VIII Workshop de Educação em Computação. Curitiba.

Brandão, L. O.; Brandão, A. A. F.; Ribeiro, R. S. (2012). "iVProg - uma ferramenta de programação visual para o ensino de algoritmos". In: Congresso Brasileiro de Informática na Educação (CBIE), 2012. Anais..., 2012.

Costa, B. D. S.; Brito, J. A.; Ribeiro, G. C.; Guedes, A. M. A. (2016). "Google Drive como Ferramenta de Acompanhamento e Avaliação na Educação de Jovens e Adultos" In: Sánchez, J. (2016) Editor. "Nuevas Ideas en Informática Educativa", Volumen 12, p. 387 - 391. Santiago de Chile.

Ellis, C.A., Gibbs, S.J. \& Rein, G.L. (1991) “Groupware - Some Issues and Experiences”. Communications of the ACM, v. 34, n. 1. 1991, New York, pp. 39-58.

Fontes, C. R. e Silva, F. W. O. (2008). "O ensino da disciplina linguagem de programação em escolas técnicas”. Ciência \& Cognição, Rio de Janeiro; v. 13, 2008, n. 2, p. 84-98, jul. 2008, ISSN 1806-5821.

Ihantola, P.; Helminen, J. e Karavirta, V. (2013). "How to study programming on mobile touch devices: Interactive python code exercises". In: Proceedings of the 13th Koli Calling International Conference on Computing Education Research. New York, NY, USA: ACM, 2013. (Koli Calling '13), p. 51-58.

Karavirta, V.; Helminen, J. e Ihantola, P. (2012). "A mobile learning application for parsons problems with automatic feedback". In: Proceedings of the 12th Koli Calling International Conference on Computing Education Research. New York, NY, USA: ACM, 2012. (Koli Calling '12), pp. 11-18.

Machado, L. D. P.; Berkenbrock, C. D. P.; Anselmo, G. e Siple, I. Z. (2018). "Uma ferramenta colaborativa para apoiar a aprendizagem de programação de computadores". Revista Brasileira de Computação Aplicada. 10. p. 23-29.

Machado, L. D. P. (2016) "Uma abordagem colaborativa para aprendizagem de programação de computadores com a utilização de dispositivos móveis". Dissertação (mestrado) - Universidade do Estado de Santa Catarina, Programa de Pós-Graduação em Computação Aplicada: Engenharia de Software. Joinville, 2016, 151p.

Moi, A; Moreira, R. C.; Moreira, P. C.; Moreira, O. C. Souza, H. C. e Kochhann, S. (2016). "A utilização da ferramenta Google Docs como recurso de ensino pela internet". AGORA Revista Eletrônica. $N^{\circ} 22.2016$.

Moura, L. (2017). "Uso do google drive na sala de aula: uma ferramenta Colaborativa para apoiar o processo de ensino-aprendizagem de produção textual no ensino médio". TCC: Licenciatura em Informática a Distância: UFRR. 
Oliveira, E. A. e Nogueira, M. (2015). “Linguagem de programação estruturada: uma reflexão sobre a didática para o ensino de programação" In: II World Congress on Systems Engineering and Information Technology. Vigo, Spain.

Oliveira, E. D. S. de; Oliveira, T. dos S.; Ferreira, P. S.; SOUSA, H. de M. e Oliveira, G. S. de. (2017). "Aprendizagem colaborativa: um experimento utilizando o Google Docs". In $23^{\circ}$ Congresso ABED de Educação a Distância. Foz do Iguaçu-PR.

Oliveira, F. N. e Moraes, D. A. F. (2013). “A utilização da tecnologia e da Internet no processo de ensino e aprendizagem da educação superior: um relato de experiência". Anais da II Jornada de Didática e I Seminário de Pesquisa do CEMAD, p. 306-320.

Pimentel, M.; Gerosa, , M. A.; Filippo, D.; Raposo, A.; Fuks, H. e Lucena, C. J. P. (2006). "Modelo 3C de Colaboração para o desenvolvimento de Sistemas Colaborativos". In Anais do III SBSC. Natal-RN, pp. 58-67.

Prensky, M. (2001). "Digital Native, Digital Immigrants”.In: Prensky, M. (2001). On the Horizon. V.9., N.5, MCB University Press.

Priesnitz Filho, W.; Abegg, I.; Simonetto, E. (2012). "Uma abordagem diferenciada no ensino de algoritmos através da utilização de uma lousa digital". GEINTEC - Gestão, Inovação e Tecnologias, 2012. v. 2, n. 2, 2012. ISSN 2237-0722.

Ramos, V. et al. (2015) “A comparação da realidade mundial do ensino de programação para iniciantes com a realidade nacional: Revisão sistemática da literatura em eventos brasileiros”. In: XXVI Simpósio Brasileiro de Informática na Educação. Maceió, AL, Brasil: [s.n.], 2015. (SBIE 2015), pp. 318-327.

Rapkiewicz, C. E. et al. (2006). "Estratégias pedagógicas no ensino de algoritmos e programação associadas ao uso de jogos educacionais". In: RENOTE - Revista Novas Tecnologias na Educação, 2006. v. 4, n. 2, 2006. ISSN 1679-1916.

Rodrigues, M. (2004). "Experiências positivas para o ensino de algoritmos". In: IV Escola Regional de Computação Bahia-Sergipe. Anais. Feira de Santana, 2004.

Salgado, N.; Castro, T.; Castro, A. (2013). “Aprendizagem colaborativa de programação com Scratch e Opensimulator". In: X Simpósio Brasileiro de Sistemas Colaborativos - SBSC 2013. [S.1.: s.n.], 2013.

Silva, T. S. C. da; Tedesco, P. C. de A. R.; Melo, J. C. B. de. (2014). "A importância da motivação dos estudantes e o uso de técnicas de engajamento para apoiar a escolha de jogos no ensino de programação". In: XXV Simpósio Brasileiro de Informática na Educação - SBIE 2014. [S.1.: s.n.], 2014. 